\title{
Observation of Generalized Optomechanical Coupling and Cooling on Cavity Resonance
}

\author{
Andreas Sawadsky, ${ }^{1}$ Henning Kaufer, ${ }^{1}$ Ramon Moghadas Nia, ${ }^{1,2}$ Sergey P. Tarabrin, ${ }^{1,3}$ \\ Farid Ya. Khalili, ${ }^{4}$ Klemens Hammerer, ${ }^{1,3}$ and Roman Schnabel ${ }^{1, *}$ \\ ${ }^{1}$ Institut für Gravitationsphysik, Leibniz Universität Hannover and Max-Planck Institut für Gravitationsphysik (Albert-Einstein Institut), \\ Callinstraße 38, D-30167 Hannover, Germany \\ ${ }^{2}$ Vienna Center for Quantum Science and Technology (VCQ), Faculty of Physics, \\ University of Vienna, Boltzmanngasse 5, 1090 Vienna, Austria \\ ${ }^{3}$ Institut für Theoretische Physik, Leibniz Universität Hannover, Appelstraße 2, D-30167 Hannover, Germany \\ ${ }^{4}$ Department of Physics, Moscow State University, Moscow 119992, Russia
}

(Received 8 September 2014; published 26 January 2015)

\begin{abstract}
Optomechanical coupling between a light field and the motion of a cavity mirror via radiation pressure plays an important role for the exploration of macroscopic quantum physics and for the detection of gravitational waves (GWs). It has been used to cool mechanical oscillators into their quantum ground states and has been considered to boost the sensitivity of GW detectors, e.g., via the optical spring effect. Here, we present the experimental characterization of generalized, that is, dispersive and dissipative, optomechanical coupling, with a macroscopic $(1.5 \mathrm{~mm})^{2}$-size silicon nitride membrane in a cavityenhanced Michelson-type interferometer. We report for the first time strong optomechanical cooling based on dissipative coupling, even on cavity resonance, in excellent agreement with theory. Our result will allow for new experimental regimes in macroscopic quantum physics and GW detection.
\end{abstract}

DOI: 10.1103/PhysRevLett.114.043601

Optomechanical cavities [1-3], whose mirrors are explicitly able to move, have been suggested to improve the sensitivity of gravitational wave detectors beyond the free-mass standard quantum limit (SQL) [4-7], to test modified models of quantum mechanics [8-13], and to realize applications in quantum information processing [1,14-16]. In such cavities, the motion of the mirror dynamically changes the cavity parameters and thus the power of the cavity field. The power change, in turn, couples back to the motion of the mirror, thereby creating a (dynamical) optomechanical coupling of the optical and mechanical degrees of freedom. Two mechanisms can be distinguished. First, the displacement of the mirror changes the resonance frequency of the cavity, leading to so-called dispersive coupling [1]. Second, the displacement of the mirror changes the linewidth of the cavity, leading to so-called dissipative coupling [17]. Up until now, optomechanics was mainly investigated in the limit of strongly dominant dispersive coupling. This regime, however, shows significant constraints. First, optical ground-state cooling [18], so far, is based on dispersive coupling, and thus requires a red-detuned light field and a cavity whose linewidth is smaller than the mechanical frequency (sideband-resolved regime) [19,20]. These requirements are unfeasible for low mechanical frequencies, i.e., in the interesting regime of macroscopic and heavy oscillators. Second, designs of next-generation gravitational wave (GW) detectors with sensitivities enhanced by the optical spring [21-23] consider only dispersive coupling so far [24]. The optical spring, however, was found to be inherently unstable [7], which results in uncontrolled
PACS numbers: $42.50 . \mathrm{Wk}, 07.10 . \mathrm{Cm}, 07.60 . \mathrm{Ly}, 42.50 . \mathrm{Ct}$ motions of the pendulum-suspended mirrors, and requires a yet-to-be-developed control system in order to exploit optomechanical effects for achieving sensitivities beyond the SQL. Generalized optomechanical systems with significant contributions from both dispersive and dissipative couplings significantly broaden the scope of optomechanics. In such systems, strong optical cooling on cavity resonance is predicted, making the sideband-resolved regime [17,25-27] unnecessary. In Refs. [27,28] it was shown that the interference of dispersive and dissipative coupling when operating close to the dark fringe can produce a stable optical spring in GW detectors. Such a setup would improve the sensitivity beyond the SQL without the need for a control system or additional light beams [29]. The application of generalized optomechanical systems is thus wide ranging and an experimental test of its mathematical description is essential. Recently, dispersive and dissipative couplings were observed with nanomechanical oscillators $[30,31]$. However, in these experiments the dissipative coupling was dominated by internal dissipation due to photon loss into unaccessible channels. Unique features such as optical cooling on resonance were not observed.

In this Letter we report on the experimental characterization of generalized optomechanical coupling in a macroscopic system of high relevance for GW detection and macroscopic quantum physics. We use a Michelson-Sagnac interferometer (MSI) with a detuned signal-recycling cavity to vary the weighting of dispersive and dissipative coupling between the light field and a silicon nitride ( $\mathrm{SiN}$ ) membrane, and compare our experimental data with the theoretical 
model. In contrast to previous works dissipative coupling in our setup is not due to internal dissipation, and in principle all photons are detectable in the output ports. For the first time, we observe optical cooling on cavity resonance providing evidence for the possibility of achieving optical cooling of massive low frequency oscillators, as well as a stable optical spring.

Our optomechanical setup is shown in Fig. 1. It represents a Michelson-Sagnac interferometer that contains a $\mathrm{SiN}$ membrane as a movable translucent mirror with a surface of $1.5 \times 1.5 \mathrm{~mm}^{2}$. The optical and optomechanical properties of this interferometer type were presented in Refs. [32-35]. In contrast to our previous realizations of the Michelson-Sagnac topology, the present work uses an unbalanced beam splitter with a reflectivity of $r_{\mathrm{BS}}^{2}=0.53$. According to theory, the unbalanced splitting is expected to increase the influence of the dissipative optomechanical coupling [27], which facilitates reaching the regime of generalized optomechanics. The movable mirror is a $\mathrm{SiN}$ membrane, which has a power reflectivity of $r_{m}^{2}=0.17$ at normal incidence at the laser wavelength of $\lambda_{0}=1064 \mathrm{~nm}$. Its subwavelength thickness of $40 \mathrm{~nm}$ results in an effective mass of $m_{\mathrm{eff}}=80 \mathrm{ng}$ for the fundamental frequency of oscillation $f_{m}=136 \mathrm{kHz}$. The membrane served as a common end mirror for both arms of the Michelson mode. Transmitted light excites the Sagnac mode. The membrane position influences the interference condition of Michelson and Sagnac modes in the interferometer output port. Assuming a perfect interferometer contrast and taking into

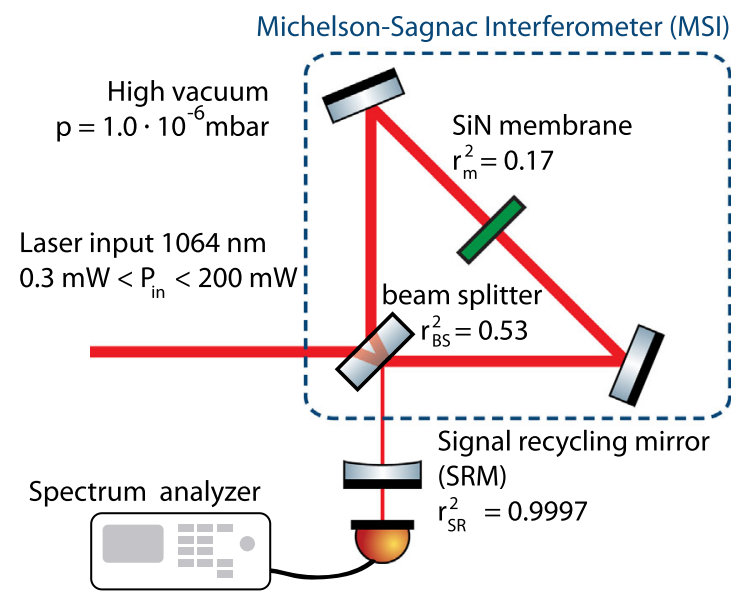

FIG. 1 (color online). Schematic of the optomechanical setup. Laser light is split into two beams, which are directed towards a translucent and partially retroreflecting membrane. Altogether four light beams, which are either reflected or transmitted through the membrane, interfere at the beam splitter, thereby forming a Michelson-Sagnac interferometer. The interferometer corresponds to a compound mirror whose effective reflectivity depends on the position of the membrane. Together with a signal-recycling mirror (SRM) in the interferometer's output port, the complete setup allows for tuning from strong dispersive to strong dissipative optomechanical coupling. account the reflectivity of the membrane, the normalized transmitted power $t_{\mathrm{MSI}}^{2}=P_{\text {out }} / P_{\text {in }}$ ranges from 0 to 0.17 [33]. The Michelson-Sagnac interferometer thus represents a compound mirror whose reflectivity ranges from $83 \%$ to $100 \%$ (assuming perfect visibility), depending on the position of the membrane.

In the present work we combined the Michelson-Sagnac interferometer with an additional mirror $\left(r_{\mathrm{SR}}^{2}=0.9997\right)$, thereby forming an optical cavity. The concept is adopted from "signal recycling" in gravitational wave detectors [36]. For the cavity-enhanced Michelson-Sagnac interferometer the transmitted power now depends on two tunable parameters: the position of the membrane and the position of the signal-recycling mirror (SRM). Its behavior is shown in Fig. 2. A shift of the position of the SRM changes the cavity resonance frequency. The displacement of the membrane also changes the cavity resonance frequency, but in particular it too changes the cavity linewidth $\gamma$ (half width at half maximum) [37]. Accordingly, the detuning
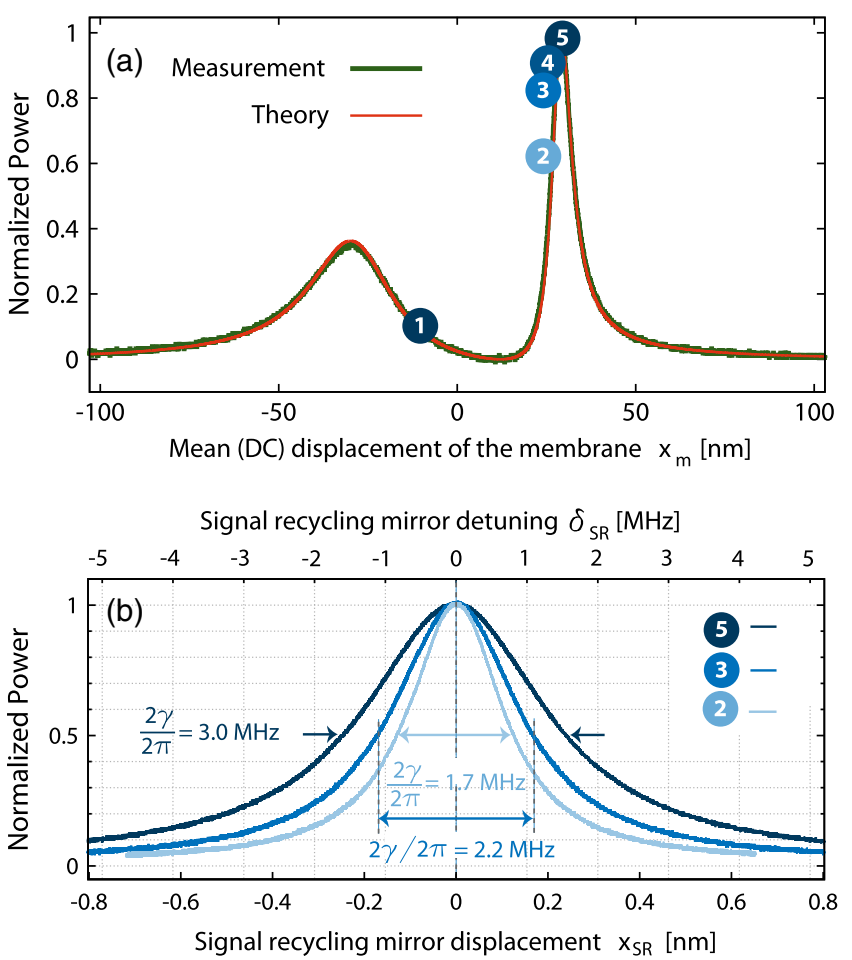

FIG. 2 (color online). Measured normalized intracavity (transmitted) light powers versus mean membrane displacement (a) and versus signal recycling mirror displacement for three membrane displacements (b). The input powers used for these measurements were 20 (a) and $5 \mathrm{~mW}$ (b), respectively. In (a) the SRM was positioned such that at operation point 5 an impedance matched resonator was achieved. Numbers 1 to 5 mark operating points of the membrane that we used for the measurements in this work. Point 1 is at $7 \%, 2$ at $60 \%, 3$ at $90 \%, 4$ at $95 \%$, and 5 at $100 \%$ of the cavity resonance peak height. Panel (b) shows the dependence of the cavity linewidth $\gamma$ on the three different chosen membrane positions 2, 3, and 5, exemplary. 
between the cavity and the laser frequency can be changed by the displacing of either the SRM or the membrane. At the membrane positions 1 to 5 the linewidth $\gamma / 2 \pi$ is tunable from 0.7 to $1.5 \mathrm{MHz}$. The cavity enhanced setup was thus far from the sideband-resolved regime, which requires $\gamma \ll 2 \pi f_{m}$, and which is necessary to reach the mechanical ground state in experiments operating in the limit of purely dispersive optomechanical coupling. In Fig. 2(b) we illustrate the linewidth measurement for membrane positions 2, 3, and 5, exemplary. For these measurements we put the membrane to the given positions and after that we scanned the position of the signal recycling mirror with a piezoactuator. To calibrate the $x$ axis in Fig. 2(b) into frequency we measured for the same membrane positions the transfer functions of the cavity with a spectrum analyzer. Together with the tunable reflectivity of the interferometer we reached a maximal finesse of about 1200. This value was not limited by the contrast when the membrane was set to maximal destructive interference at the output port but by losses in optical components $(0.5 \%$ in total). The effective cavity length resulted in a free-spectral range of FSR $=c /(2 \mathcal{L})=1.7 \mathrm{GHz}$ with an effective cavity length of $2 \mathcal{L}=0.174 \mathrm{~m}$. The complete interferometer according to Fig. 1 was set up in a highvacuum environment $\left(p=1.0 \times 10^{-6}\right.$ mbar $)$ to avoid damping of the oscillator motion by residual gas. In the absence of the SRM (i.e., with neither optical cooling nor heating) the oscillator mechanical quality factor $Q$ of the fundamental oscillation mode was determined by ring-down measurements to be $Q_{\text {inital }}=5.8 \times 10^{5}$.

The generalized optomechanical coupling, which includes dispersive and dissipative coupling, was observed by detecting the interferometer's output power spectrum with a photodiode. Figure 3 shows two example spectra. The peaks correspond to the thermally excited motion of the membrane's fundamental resonance. Both measurements were recorded when the membrane position was set such that the carrier light interfered almost destructively in the interferometer output port (position 1 in Fig. 2). The measurement in Fig. 3(a) was performed without a SRM and calibrated using the method of Ref. [32], whereas Fig. 3(b) refers to a measurement with a (detuned) signal recycling cavity. The latter shows strong damping (optical cooling) of the membrane oscillation. To derive the damped $Q$ factor we fitted the $Q_{\text {eff }}$ value such that our model well described the height and the width of the thermally excited membrane resonance [38]. $Q_{\text {eff }}$ here is the resulting $Q$ factor of the membrane, which was influenced by the radiation pressure force (due to both dispersive and dissipative coupling). This adds an optically induced damping, which changes the $Q$ factor, which is modeled in Ref. [27] and summarized in the Supplemental Material [37].

To observe generalized optomechanical coupling we exploited the tunability of dissipative and dispersive coupling in our setup, and we quantified the optical cooling at

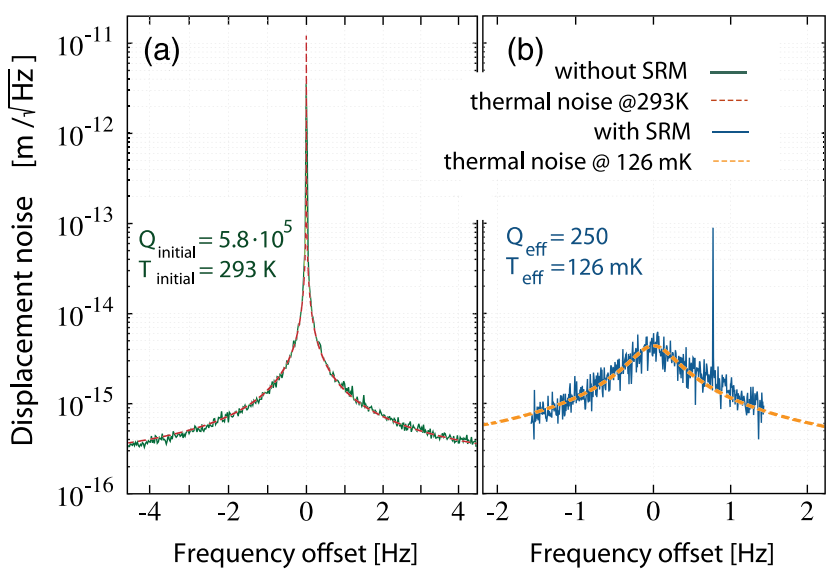

FIG. 3 (color online). Interferometer output spectra (a) without the SRM (i.e., without the cavity) close to the dark fringe, and (b) for a cavity-enhanced interferometer. The latter spectrum was taken at operation point 1 [Fig. 2(a)], with a detuning for which our theory predicts a particularly strong cooling effect. The input power was $200 \mathrm{~mW}$ in (a) and (b). The thermal noise levels (dashed lines) represent fits to the measurements with the mechanical $Q_{\text {eff }}$ factor as the fitting parameter. In this measurement we could reach an effective mechanical quality factor $Q_{\text {eff }}=250$ and an effective temperature of $T_{\text {eff }}=126 \mathrm{mK}$.

various positions of the membrane and SRM, see Fig. 4. The power inside the cavity was sufficiently low to not influence the measured quality factors by optical absorption of the membrane. In all measurements we positioned the membrane in the vicinity of a standing-wave node rather than in the vicinity of a standing-wave antinode [33]. The frequency shift of the membrane resonance due to optical absorption was confirmed to be always less than $500 \mathrm{~Hz}$. In this case the influence on the $Q$ factor is estimated to be on the order of a few percent and thus negligible for our analysis presented here.

Figure 4 represents the observation of generalized optomechanical coupling, i.e., strong signatures of interfering dispersive and dissipative couplings. All four graphs are distinct from conventional optomechanics with strongly dominating dispersive coupling. The first graph corresponds to membrane position 2 in Fig. 2. For negative detuning of the signal-recycling cavity we observed optical cooling $\left(Q_{\text {eff }}<Q_{\text {inital }}\right)$, similar to the purely dispersive regime. But the same graph also shows optical cooling on cavity resonance (zero detuning). This effect is not possible in dispersive optomechanics. For larger positive detunings the membrane oscillation is parametrically heated $\left(Q_{\text {eff }}>Q_{\text {inital }}\right)$. This effect is intrinsically unstable and eventually damped by the nonlinear behavior of the membrane for strong oscillations. Instability regions are marked as yellow areas. In the second graph optical cooling on resonance is more pronounced, again in excellent agreement with our theory (solid line) [27,37]. The third graph shows a further evolution of the cooling spectrum. The optical cooling is observed up to detunings as large as 


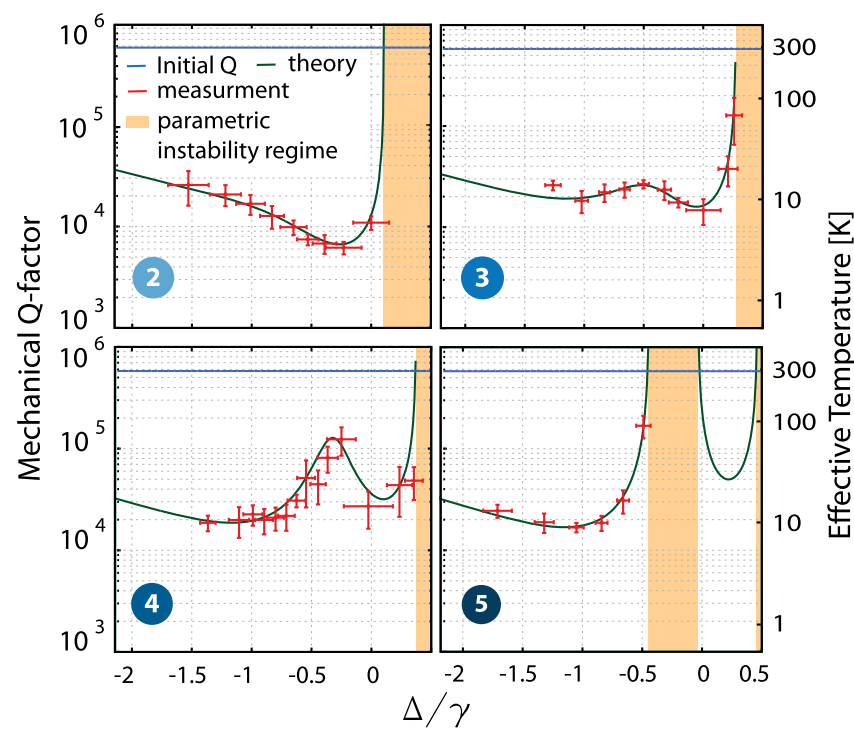

FIG. 4 (color online). Observed evidence of generalized optomechanical coupling and cooling on cavity resonance. We measured the effective mechanical $Q$ factor versus cavity detuning for four membrane positions as given in Fig. 2(a) with an input power of $20 \mathrm{~mW}$. Already in the first graph cooling on cavity resonance is clearly visible. The next panels show an increasing influence of dissipative coupling and cooling regions that significantly expand into the region of positive (blue) detunings. The last panel confirms the theoretically predicted existence of a new instability region, which appears at negative (red) detunings. Solid lines refer to our theory of generalized optomechanical coupling. Regions of instability are marked yellow. The error bars represent the standard deviation of five independent measurements.

$\Delta / \gamma=0.3$. The last graph eventually shows the occurrence of a new instability region for small negative detunings. The newly appearing instability region is already visible as a reduced cooling performance in subfigures 3 and 4 . Our theory predicts a well-separated cooling region at positive detunings, which, however, could not be explored in the present experiment [39]. The observed behavior of the $Q$ factor is due to a complex structure of the radiation pressure noise spectral density: in the case of generalized optomechanical coupling this spectral density is a mixture of a Lorentz profile and a Fano profile corresponding to the dispersive and dissipative contributions, respectively $[17,25,27,37]$. Figure 5 shows optical cooling on resonance (measured at membrane position 3) versus input power. Again, we find compelling agreement with the model summarized in the Supplemental Material [37].

In conclusion, we realized a cavity-optomechanical setup with strong dissipative coupling between the cavity field and the mechanical oscillator, which did not rely on photon loss into unaccessible channels, in contrast to the experiments reported in Refs. [30,31]. In the language of the Hamiltonian description developed in Refs. [17,25,40] dispersive and dissipative coupling strengths can be

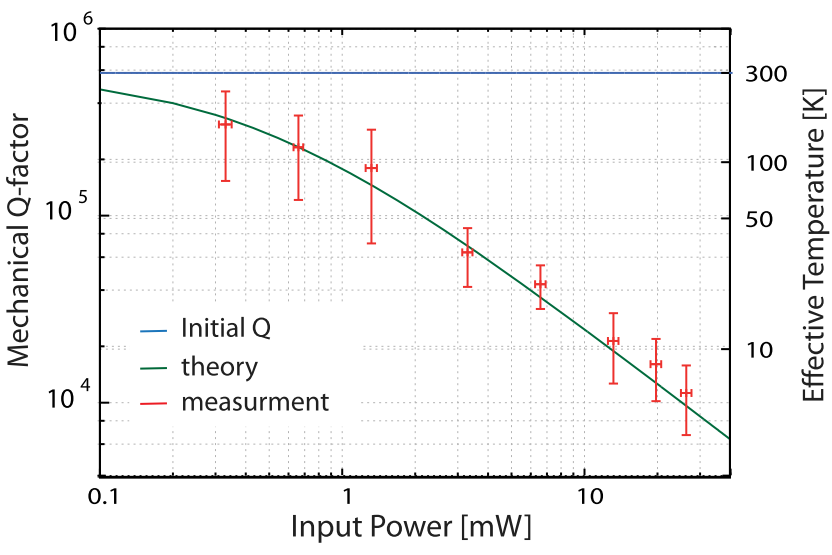

FIG. 5 (color online). Cooling on cavity resonance versus input power. The measurements are in excellent agreement with the theoretical model presented in Refs. [27,37]. Here, the membrane was at position 3, as described in Fig. 2.

characterized, respectively, by rates (per single photon) $g_{\omega}=$ $x_{\mathrm{ZPF}} d \omega_{\mathrm{c}}(x) /\left.d x\right|_{x_{0}}$ and $g_{\gamma}=x_{\mathrm{ZPF}} d \gamma(x) /\left.d x\right|_{x_{0}}$, where $x_{\mathrm{ZPF}}=$ $\sqrt{\hbar /\left(2 m \omega_{\mathrm{m}}\right)}$ is the amplitude of zero-point mechanical fluctuations of the membrane, $\omega_{\mathrm{c}}(x)$ and $\gamma(x)$ are the position-dependent cavity eigenfrequency and half linewidth, respectively, and $x_{0}$ is the mean $(\mathrm{dc})$ position of the membrane. Explicit formulas for the coupling rates can be found in the Supplemental Material [37]. For the parameters of the present setup these rates are tunable from 0 to about $0.1 \mathrm{~Hz}$, depending on the exact position of the membrane.

For the first time optical cooling of a mechanical oscillator through dissipative coupling, including, in particular, cooling on cavity resonance, was observed. We measured a strong reduction in effective temperature of 3 orders of magnitude. A reduction of the mechanical quality factor on resonance, as well as the existence of a second instability on the cooling side of the cavity resonance, are key predictions for a dissipative coupling in our experiment. We found excellent agreement with our model for generalized optomechanical coupling. Stronger cooling is predicted if the internal loss of the interferometer can be reduced. Ground state cooling is predicted outside the sideband-resolved regime [25]. Our work might pave the way towards ground-state cooling of heavy objects. Since the dissipative coupling in our setup was external, the information gathered by photodiodes can in principle be used for conditionally defining an almost pure mechanical quantum state as suggested in Refs. [41,42] allowing for quantum physics with the motion of heavy objects.

Overall, we confirmed the theory of generalized optomechanical coupling in a regime that is of interest also in the field of gravitational wave detection. Dissipative coupling can give rise to a stable optical spring (that is a positive shift of the mechanical frequency and damping) [27], as proposed in Ref. [28] for the improvement of GW 
detectors. The effects of a stable spring can in principle also be tested in our setup. The expected frequency increase due to an optical spring in our current setup, however, is small and masked by frequency changes due to absorption, i.e., absorptive heating.

This work was supported by the Marie Curie Initial Training Network cQOM, by the ERC Advanced Grant MassQ, and by the International Max Planck Research School for Gravitational Wave Astronomy (IMPRS). H. K. acknowledges support from the HALOSTAR scholarship program. K. H. and S. P. T. acknowledge support through EU project iQUOEMS. The work of F. Khalili was supported by LIGO NSF Grant No. PHY-1305863 and Russian Foundation for Basic Research Grant No. 11-02-00383-a.

* Corresponding author. roman.schnabel@aei.mpg.de

[1] M. Aspelmeyer, T. J. Kippenberg, and F. Marquardt, Rev. Mod. Phys. 86 (2014).

[2] Y. Chen, J. Phys. B 46, 104001 (2013).

[3] P. Meystre, Ann. Phys. (Berlin) 525, 215 (2013).

[4] V. B. Braginsky and A. B. Manukin, Sov. Phys. JETP 25, 653 (1967).

[5] V. B. Braginsky, A. B. Manukin, and M. Yu. Tikhonov, Sov. Phys. JETP 31, 829 (1970).

[6] V. B. Braginsky, M. L. Gorodetsky, and F. Y. Khalili, Phys. Lett. A 232, 340 (1997).

[7] A. Buonanno and Y. Chen, Phys. Rev. D 65, 042001 (2002).

[8] M. Bahrami, M. Paternostro, A. Bassi, and H. Ulbricht, Phys. Rev. Lett. 112, 210404 (2014).

[9] J. Bateman, S. Nimmrichter, K. Hornberger, and H. Ulbricht, Nat. Commun. 5, 4788 (2014).

[10] W. Marshall, C. Simon, R. Penrose, and D. Bouwmeester, Phys. Rev. Lett. 91, 130401 (2003).

[11] S. Nimmrichter, K. Hornberger, P. Haslinger, and M. Arndt, Phys. Rev. A 83, 043621 (2011).

[12] S. Nimmrichter, K. Hornberger, and K. Hammerer, Phys. Rev. Lett. 113, 020405 (2014).

[13] O. Romero-Isart, A. C. Pflanzer, F. Blaser, R. Kaltenbaek, N. Kiesel, M. Aspelmeyer, and J. I. Cirac, Phys. Rev. Lett. 107, 020405 (2011).

[14] S. Rips and M. J. Hartmann, Phys. Rev. Lett. 110, 120503 (2013).

[15] K. Stannigel, P. Rabl, A. S. Sørensen, P. Zoller, and M. D. Lukin, Phys. Rev. Lett. 105, 220501 (2010).

[16] K. Stannigel, P. Rabl, A. S. Sørensen, M. D. Lukin, and P. Zoller, Phys. Rev. A 84, 042341 (2011).

[17] F. Elste, S. M. Girvin, and A. A. Clerk, Phys. Rev. Lett. 102, 207209 (2009).

[18] J. Chan, T. P. M. Alegre, A. H. Safavi-Naeini, J. T. Hill, A. Krause, S. Gröblacher, M. Aspelmeyer, and O. Painter, Nature (London) 478, 89 (2011).
[19] F. Marquardt, J. P. Chen, A. A. Clerk, and S. M. Girvin, Phys. Rev. Lett. 99, 093902 (2007).

[20] I. Wilson-Rae, N. Nooshi, W. Zwerger, and T. J. Kippenberg, Phys. Rev. Lett. 99, 093901 (2007).

[21] G. M. Harry, Classical Quantum Gravity 27, 084006 (2010).

[22] T. Accadia et al., Classical Quantum Gravity 28, 114002 (2011).

[23] K. Somiya, Classical Quantum Gravity 29, 124007 (2012).

[24] A. Buonanno and Y. Chen, Phys. Rev. D 67, 062002 (2003).

[25] A. Xuereb, R. Schnabel, and K. Hammerer, Phys. Rev. Lett. 107, 213604 (2011).

[26] T. Weiss and A. Nunnenkamp, Phys. Rev. A 88, 023850 (2013).

[27] S. P. Tarabrin, H. Kaufer, F. Y. Khalili, R. Schnabel, and K. Hammerer, Phys. Rev. A 88, 023809 (2013).

[28] N. Vostrosablin and S. P. Vyatchanin, Phys. Rev. D 89, 062005 (2014).

[29] H. Rehbein, H. Müller-Ebhardt, K. Somiya, S. L. Danilishin, R. Schnabel, K. Danzmann, and Y. Chen, Phys. Rev. D 78, 062003 (2008).

[30] M. Li, W. H. P. Pernice, and H. X. Tang, Phys. Rev. Lett. 103, 223901 (2009).

[31] M. Wu, A. C. Hryciw, C. Healey, D. P. Lake, H. Jayakumar, M. R. Freeman, J. P. Davis, and P. E. Barclay, Phys. Rev. X 4, 021052 (2014).

[32] T. Westphal, D. Friedrich, H. Kaufer, K. Yamamoto, S. Goßler, H. Müller-Ebhardt, S. L. Danilishin, F. Y. Khalili, K. Danzmann, and R. Schnabel, Phys. Rev. A 85, 063806 (2012).

[33] D. Friedrich, H. Kaufer, T. Westphal, K. Yamamoto, A. Sawadsky, F. Y. Khalili, S. L. Danilishin, S. Goßler, K. Danzmann, and R. Schnabel, New J. Phys. 13, 093017 (2011).

[34] H. Kaufer, A. Sawadsky, T. Westphal, D. Friedrich, and R. Schnabel, New J. Phys. 14, 095018 (2012).

[35] H. Kaufer, Ph.D. thesis, Leibniz Universität Hannover, 2013.

[36] B. J. Meers, Phys. Rev. D 38, 2317 (1988).

[37] See Supplemental Material at http://link.aps.org/ supplemental/10.1103/PhysRevLett.114.043601 for calculations.

[38] K. Yamamoto, D. Friedrich, T. Westphal, S. Goßler, K. Danzmann, K. Somiya, S. L. Danilishin, and R. Schnabel, Phys. Rev. A 81, 033849 (2010).

[39] The problem is that by passing through a region of instability the membrane is excited to very large amplitudes that cover the full range of displacements shown in Fig. 2(a). Our linearized model and its predictions regarding (in) stabilities is not valid for such large amplitudes.

[40] T. Weiss, C. Bruder, and A. Nunnenkamp, New J. Phys. 15, 045017 (2013).

[41] H. Müller-Ebhardt, H. Rehbein, R. Schnabel, K. Danzmann, and Y. Chen, Phys. Rev. Lett. 100, 013601 (2008).

[42] H. Müller-Ebhardt, H. Rehbein, C. Li, Y. Mino, K. Somiya, R. Schnabel, K. Danzmann, and Y. Chen, Phys. Rev. A 80, 043802 (2009). 